\title{
Using postoperative SNOT-22 to help predict the probability of revision sinus surgery*
}

\author{
Luke Rudmik', Zachary M. Soler², Claire Hopkins³ \\ Rhinology 54: 111-116, 2016 \\ DOI:10.4193/Rhino15.284 \\ ' Division of Otolaryngology - Head and Neck Surgery, Department of Surgery, University of Calgary, Calgary, AB, Canada \\ *Received for publication: \\ 2 Division of Rhinology and Sinus Surgery, Department of Otolaryngology - Head and Neck Surgery, Medical University of South Caro- September 15, 2015 \\ lina, Charleston, SC, USA \\ Accepted: October 22, 2015
}

${ }^{3}$ Department of ENT, Guy's and St Thomas' NHS Trust, London, SE1 9RT United Kingdom

\begin{abstract}
Background: There is a need to develop a patient-level strategy to identify those at higher risk of requiring revision ESS since this may assist clinicians in tailoring their postoperative management. This study evaluated whether identifying changes in the postoperative 22-item Sinonasal Outcome Test (SNOT-22) can help identify patients at increased risk of needing revision sinus surgery for refractory chronic rhinosinusitis (CRS).
\end{abstract}

Methods: 668 CRS patients undergoing primary ESS with complete 60-month follow-up were evaluated in this prospective, longitudinal cohort study. Outcomes were evaluated in an unselected cohort and a 'low-risk' cohort, which was comprised of patients without a history of asthma or aspirin sensitivity.

Results: Failing to achieve an improvement of greater than one minimal clinically important difference (MCID; 9 points) at 3 months after primary ESS and a deterioration of greater than one MCID (ie. > 9 points) from the 3- to 12-month follow-up periods was associated with an increased risk of revision ESS in both the unselected and 'low-risk' CRS cohorts.

Conclusion: Outcomes from this study suggest that identifying MCID changes in the SNOT-22 score within 12 months after primary ESS can identify patients at increased risk for needing revision surgery.

Key words: chronic rhinosinusitis, endoscopic sinus surgery, revision surgery, SNOT-22, patient-centered care

\section{Introduction}

When indicated, revision endoscopic sinus surgery (ESS) for chronic rhinosinusitis (CRS) has been shown to improve clinical outcomes ${ }^{(1-5)}$. However, the risk of requiring revision ESS represents a major concern to patients ${ }^{(6)}$ while creating an additional and potentially avoidable cost to the health care system. Therefore, developing simple clinical strategies to identify patients who are at risk for progressing to require revision ESS would not only improve patient-centered care but may also help clinicians modify their management to reduce the need for a revision surgery.
On an aggregate CRS scale, several studies have quantified the 5 -year risk of requiring revision ESS to be between $10 \%$ and $20 \%{ }^{(7-11)}$, while the presence of certain comorbidities such as asthma and aspirin sensitivity, along with other factors such as having high baseline computed tomography (CT) staging ${ }^{(12)}$ or incomplete sinus dissection ${ }^{(13)}$ have been associated with elevated revision rates between $25 \%$ and $40 \%{ }^{(8-10,12)}$. Other high risk factors for revision ESS include predominant Th2 type inflammation ${ }^{(14)}$ and high tissue eosinophilia ${ }^{(15,16)}$. However, despite the presence of known risk factors for revision surgery, evidence for several of these clinical characteristics have failed to reliably predict revision $\operatorname{ESS}^{(3,17,18)}$ and the immunologic measures can 
be difficult to standardize and interpret for clinical use. Furthermore, there is a large cohort of patients who lack these traditional high-risk characteristics who end up progressing to need revision ESS. Developing a more reliable and simple method to identify patients who are at risk for progressing to need revision may help the clinician tailor their management and provide more aggressive medical therapies or increase post-operative surveillance to potentially avoid another sinus surgery.

The objective of this study is to evaluate whether the 22-item Sinonasal Outcome Test ${ }^{(19)}$ (SNOT-22) can function to help identify the probability of revision surgery in patients undergoing primary ESS for refractory CRS.

\section{Materials and methods}

This is a prospective longitudinal observational cohort study that is part of the United Kingdom (UK) National Comparative Audit of Surgery for Nasal Polyposis and Chronic Rhinosinusitis ${ }^{(8)}$. The full methodology for the UK audit has been previously reported ${ }^{(7)}$, but, in summary, data was prospectively collected on all consecutive patients undergoing ESS in 80 UK Hospital Trusts, under the care of 298 consultant Otolaryngology (ENT) surgeons. Data was collected at baseline, and then postoperatively at 3-, 12-, 36-, and 60-months after ESS. Questionnaires were mailed to participants from the study coordinator with a reply paid envelope, and therefore ongoing follow-up was not determined by clinical need as assessed by the operating surgeon.

For this study, we excluded patients with a history of ESS before the index case reported in the UK audit and patients without data collected at each of the post-operative follow-up time periods. The primary outcome measure was the SNOT-22 and secondary outcome was the need for revision surgery within the study period as reported by the patient on their final follow-up questionnaire at 60 months. The stability of the SNOT-22 at each time point was assessed as a population mean for all respondents at each time point. To evaluate the risk of revision ESS in 'low-risk' patients undergoing primary ESS, we excluded those with co-morbid asthma and aspirin sensitivity and repeated the analysis.

All patients included in this study were dichotomized according to whether or not they had achieved an improvement in SNOT22 score greater than the MCID (9 points) ${ }^{(19)}$ at each follow-up point, compared to their baseline score. Two postoperative scenarios involving MCID changes in SNOT-22 were evaluated to determine if they were independent predictors for revision surgery by 60 months: 1) failing to achieve an immediate (ie. 3 month follow-up) post-operative MCID improvement, and 2) a deterioration of one MCID from 3- to 12-months after primary ESS.
Table 1. Patient demographics.

\begin{tabular}{|lc|}
\hline Age & Mean (SD) \\
\hline Pre-operative SNOT-22 & $52.0(13.5)$ \\
\hline Pre-operative Lund-Mackay score & $39.4(19.3)$ \\
\hline Male & $9.7(6.4)$ \\
\hline Asthmatic & $\mathbf{n}(\%)$ \\
\hline Aspirin Sensitivity & $380(56.9)$ \\
\hline Nasal polyps & $179(26.8)$ \\
\hline
\end{tabular}

SNOT, sinonasal outcome test; SD, standard deviation; $n$, number.

\section{Statistical analysis}

The association between achieving the MCID improvement and revision surgery was tested using Chi-squared $\left(\chi^{2}\right)$ probability tests. Multivariable logistic regression was performed and odds ratios (ORs) reported to identify whether or not several patient variables were independent predictors for revision ESS.

\section{Results}

Complete data at every time point was available for 668 patients undergoing primary ESS. Figure 1 demonstrates study recruitment along with subsequent attrition due to loss to follow-up and application of study exclusion criteria. We have previously shown that there were no significant baseline differences between responders and those patients lost to follow-up ${ }^{(8)}$.

Patient demographics are shown in Table 1. Mean revision rate for the entire cohort was $8.5 \%$ at 60 months. At 3 months after ESS, $70.1 \%$ of patients achieved a MCID improvement in SNOT22 scores, compared to their pre-operative baseline. 29 patients (4.3\%) had a baseline SNOT-22 score of less than 10 , who by definition are unable to achieve a reduction of greater than the MCID, and a further 84 (12.6\%) had a score of between 10 and 20. The proportion of MCID improvement declined at each time point after ESS, and by 60 months only $57.5 \%$ of patients reported a MCID improvement compared to their baseline score. There was a statistically significant association between not having a MCID improvement at 3-, 12-, and 36-months and increased risk of needing revision surgery (Table 2 ). This indicates that failing to maintain a MCID improvement at 3-, 12-, and 36-months, compared to the preoperative SNOT-22 score, increases the risk of progressing to revision ESS.

Unselected Cohort: Predictive Value of Postoperative SNOT22 for Revision ESS

A total of 170 patients (26\%) patients who had initially reported 


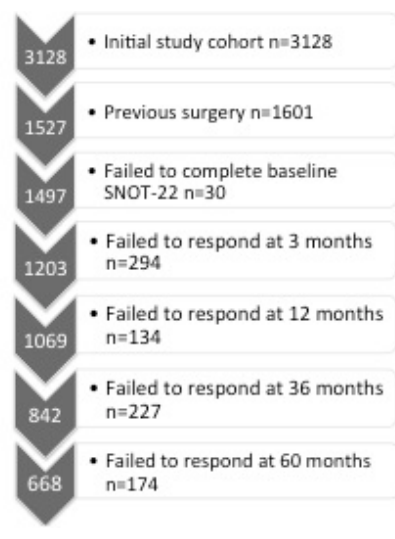

Figure 1. Flow diagram of patient recruitment and final study cohort.

a MCID improvement at 3-months reported a deterioration of at least one MCID (ie. 9 points) from the 3- to 12-month SNOT22 score. Deterioration in the SNOT-22 score greater than one MCID (ie. 9 points) from the 3- to 12 month SNOT-22 score after primary ESS was found to be a significant independent risk factor for revision surgery (OR 2.7 ( $95 \% \mathrm{Cl} 1.3-5.9) ; \mathrm{p}=0.01)$. This indicates that even though a patient may have an overall MCID improvement at 12 months, compared to their preoperative baseline SNOT-22 score, they are at higher risk for progressing to need revision ESS if they demonstrate a 9-point deterioration in SNOT-22 score at 12 months compared to their 3-month score.

We have previously shown that the chance of achieving an improvement of greater than the MCID is directly correlated to the baseline score; indeed those with a score of less than 10 cannot achieve the MCID. However, the multivariable regression suggests that beyond the low SNOT-22 baseline group, there is little additional risk of revision surgery associated with increasing baseline SNOT-22 (OR 1.01, p=0.049). In contrast, failing to achieve an improvement of greater than one MCID (ie. 9 points) at 3 months after primary ESS, even after the baseline SNOT-22 in controlled for within the regression, was associated with a greatly increased risk of revision surgery within 60 months after primary surgery (OR 4.3 ( $95 \% \mathrm{Cl} 1.93-9.66) ; \mathrm{p}<0.001)$. Asthma and aspirin sensitivity were other independent risk factors for needing revision surgery (all $\mathrm{p}<0.05$ ). Age, gender, polyp status, and Lund-MacKay CT score were not found to be risk factors for revision surgery within 60 months (Table 3 ).

'Low-Risk' Cohort: Predictive Value of Postoperative SNOT22 for Revision

After excluding patients with asthma and aspirin sensitivity ( $n=184)$, the multivariate regression analysis was repeated for the 'low-risk' cohort $(n=484)$. A deterioration of greater than one MCID from the 3- to 12 month SNOT-22 score after primary ESS remained a significant risk factor for revision surgery within
Table 2. Association between achieving an MCID improvement and revision $\mathrm{ESS}, \mathrm{n}=668$.

\begin{tabular}{|c|c|c|c|c|}
\hline $\begin{array}{c}\text { Time after } \\
\text { Primary } \\
\text { ESS* }\end{array}$ & \multicolumn{2}{|c|}{$\begin{array}{l}\text { Achieved a MCID } \\
\text { improvement }\end{array}$} & $\begin{array}{c}\text { Revision ESS } \\
\text { within } 60 \text { months } \\
\text { after primary ESS } \\
\text { (\%) }\end{array}$ & \\
\hline \multirow[t]{2}{*}{3 months } & Yes & $\begin{array}{l}n=468 \\
(70.1 \%)\end{array}$ & $\mathrm{n}=34(7.3 \%)$ & \multirow[t]{2}{*}{$p=0.018$} \\
\hline & No & $n=200$ & $n=26(13.0 \%)$ & \\
\hline \multirow[t]{2}{*}{12 months } & Yes & $\begin{array}{c}n=425 \\
(63.6 \%)\end{array}$ & $n=25(5.9 \%)$ & \multirow{2}{*}{$p<0.001$} \\
\hline & No & $n=243$ & $\mathrm{n}=35(14.4 \%)$ & \\
\hline \multirow[t]{2}{*}{36 months } & Yes & $\begin{array}{l}n=407 \\
(60.9 \%)\end{array}$ & $n=27(6.6 \%)$ & \multirow[t]{2}{*}{$p=0.008$} \\
\hline & No & $n=261$ & $n=33(12.6 \%)$ & \\
\hline \multirow[t]{2}{*}{60 months } & Yes & $\begin{array}{c}n=384 \\
(57.5 \%)\end{array}$ & $n=33(8.6 \%)$ & \multirow[t]{2}{*}{$p=0.683$} \\
\hline & No & $n=284$ & $n=27(9.5 \%)$ & \\
\hline
\end{tabular}

ESS, endoscopic sinus surgery; MCID, minimal clinically important difference; $n$, number.

60 months after primary surgery (OR $3.2(95 \% \mathrm{Cl} 1.15-8.65)$; $\mathrm{p}=0.026$ ). Failing to achieve an MCID improvement at 3 months after primary ESS remained associated with an increased risk of revision ESS within 60 months after primary surgery (OR 2.9 (95\% Cl 1.15- 8.64); $p=0.038$ )(Table 4).

Using the deterioration of one MCID from the 3- to 12-month SNOT-22 score after primary ESS had a sensitivity of $42.8 \%$ and

Table 3. Predictors of revision ESS in unselected CRS cohort.

\begin{tabular}{|c|c|c|}
\hline Variable & OR $(95 \% \mathrm{CI})$ & p-value \\
\hline Baseline SNOT-22 score & $1.01(1-.00-1.03)$ & 0.049 \\
\hline $\begin{array}{l}\text { Failure to achieve MCID improve- } \\
\text { ment at } 3 \text { months after primary ESS }\end{array}$ & $4.3(1.9-9.7)$ & $<0.001$ \\
\hline $\begin{array}{l}\text { Deterioration of one MCID from } 3 \text { - } \\
\text { to } 12 \text {-months after primary ESS }\end{array}$ & $2.7(1.2-5.8)$ & 0.01 \\
\hline Asthma & $2.8(1.3-5.9)$ & 0.008 \\
\hline Aspirin sensitivity & $5.4(1.2-23.9)$ & 0.03 \\
\hline Lund-MacKay CT score & $1.05(0.98-1.12)$ & 0.11 \\
\hline Gender (male) & $0.6(0.3-1.3)$ & 0.19 \\
\hline Age & $1.0(0.96-1.01)$ & 0.21 \\
\hline Presence of nasal polyp & $1.2(0.5-2.9)$ & 0.69 \\
\hline
\end{tabular}

CRS, chronic rhinosinusitis; MCID, minimal clinically important difference; ESS, endoscopic sinus surgery; OR, odds ratio; $\mathrm{CT}$, computed tomography. 
Table 4. Predictors of Revision ESS in 'Low-risk' patients with CRS.

\begin{tabular}{|lcc|}
\multicolumn{1}{c}{ Variable } & OR $(95 \% \mathrm{CI})$ & p-value \\
\hline Baseline SNOT-22 score & $1.01(0.99-1.04)$ & 0.14 \\
$\begin{array}{l}\text { Failure to achieve MCID improve- } \\
\text { ment at 3 months after primary ESS }\end{array}$ & $3.6(1.24-10.44)$ & 0.018 \\
\hline $\begin{array}{l}\text { Deterioration of one MCID from 3- } \\
\text { to 12-months after primary ESS }\end{array}$ & $2.7(1.2-7.3)$ & 0.048 \\
\hline Lund-MacKay CT score & $1.1(0.96-1.16)$ & 0.234 \\
\hline Gender (male) & $0.5(0.17-1.57)$ & 0.243 \\
\hline Age & $1.0(0.93-1.01)$ & 0.151 \\
\hline Presence of nasal polyp & $1.1(0.32-3.78)$ & 0.87
\end{tabular}

CRS, chronic rhinosinusitis; MCID, minimal clinically important difference; ESS, endoscopic sinus surgery; OR, odds ratio; $C T$, computed tomography.

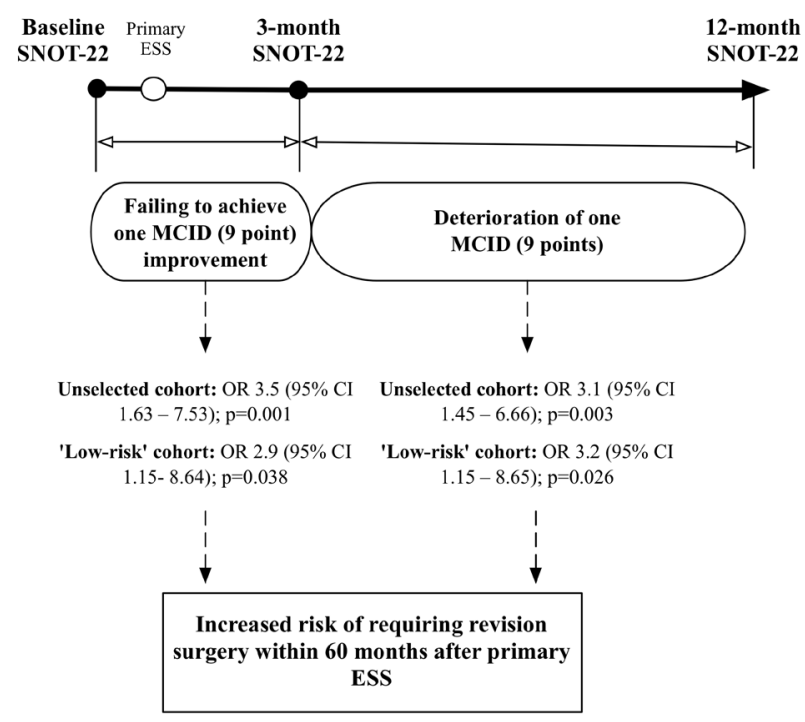

Figure 2. Postoperative SNOT-22 helping to predict the risk of revision ESS. a specificity of $76.5 \%$ in predicting the need for revision surgery within 60 months after surgery.

\section{Discussion}

Outcomes from this study suggest that measuring and comparing SNOT-22 scores at baseline and then at 3 and 12 months after primary ESS may assist in identifying patients at increased risk for needing revision surgery. Failure to achieve an immediate MCID (ie. > 9 points) improvement at 3-months and a deterioration of greater than one SNOT-22 MCID from the 3-month to 12-month SNOT-22 score were both associated with an increased risk of revision ESS in both the unselected cohort and 'low-risk' cohort of patients with CRS (Figure 2). Therefore, in the absence of traditional high-risk characteristics for revision ESS, such as asthma and aspirin sensitivity, a clinician may identify patients at risk for progressing to revision ESS by monitoring the changes in SNOT-22 scores within the first 12 months after ESS. Future research will need to elucidate whether postoperative treatment strategies can reduce the risk of needing revision ESS in patients who suffer a worsening of their SNOT-22 of greater than one MCID at 12 months.

In times of global austerity measures, there is a drive to reduce the costs of health-care provision. One way to reduce costs is to reduce unwarranted or avoidable surgical interventions and postoperative follow-ups. In the UK, a state-funded healthcare system, many clinical commissioning groups will not pay for more than one post-operative follow-up appointment, necessitating early discharge after surgery. Many private medical insurance companies are taking a similar approach. Excluding any debate regarding the need for early post-operative debridement, it would seem reasonable, given the stability of the mean population SNOT-22 scores, to discharge patients who have derived a MCID improvement in SNOT-22 score at 3 to 6 months post-surgery. However, it must be remembered that these are population means, and they do not necessarily reflect what is happening at an individual patient level.

In this large population-based observational study, $70 \%$ of patients undergoing primary ESS received an MCID improvement at 3-months which is similar to a North American-based study which demonstrated approximately $80 \%$ of patients achieved an MCID improvement after ESS ${ }^{(20)}$. This supports the notion that in the appropriately selected patients with CRS, patients should be informed that they have an estimated $70 \%$ to $80 \%$ chance of obtaining a clinically meaningful improvement in their quality of life after primary ESS. Furthermore, after an initial MCID improvement in SNOT-22 at 3 months after primary ESS, $26 \%$ of both the unselected and 'low-risk' cohorts reported a deterioration of greater than one MCID from the 3- to 12-month scores, and this was associated with an increased risk of progressing to revision ESS within 60 months. This suggests that stability of the population mean over the long-term follow-up may mask changes at individual patient-level, and supports the need to measure patient-reported outcomes to at least 12 months after primary ESS. We appreciate that due to financial constraints along with the effect of patient migration with loss to follow-up, that extending patient monitoring beyond 12 months in research trials can be challenging, but linkage to electronic health records beyond this point may be a cost-effective means to achieve longer-term follow-up on a health care system level.

Looking at both the unselected and 'low risk' CRS cohorts, a failure to achieve an improvement of greater than one MCID 
at 3 months after ESS was also an independent predictor for the need of revision surgery. The failure to achieve a MCID at 3-months may suggest that residual disease is present due to inadequate surgery or the patient did not require ESS and hence unwarranted surgery was performed. In contrast, deterioration after an initial improvement at 3 months is likely related to poorly controlled inflammatory disease and may prompt more intensive medical therapy in the hope of preventing disease recurrence. With the goal to improve clinical care, when patients are identified to be at higher risk of needing a revision surgery using outcomes from this study, future research will need to evaluate whether or not intervening with more intensive postoperative surveillance or improved postoperative medical therapy ( \pm rescue medications) can prevent recurrence of symptoms requiring revision surgery.

Given that $8.5 \%$ of patients with CRS who received primary ESS in this study required a revision surgery within 60 months, it would be preferable to identify those who are known to be at higher risk of revision prior to discharging them from the care of an Otolaryngologist. This might allow the higher-risk sub-group to be reviewed in person, or monitored remotely using electronic completion of SNOT-22 scores to prompt recall. In agreement with the published literature, we found that asthma and aspirin sensitivity to be risk factors for revision ESS. However, given that a large proportion of CRS patients undergoing primary ESS lack these traditional risk factors, monitoring changes in the SNOT-22 score between 3 and 12-months after surgery can help identify 'low-risk' patients who are at increased risk for revision ESS. With this data in mind, clinicians should consider recording sinusspecific QOL scores in a longitudinal fashion prior to surgery and then again at 3 and 12 months after surgery. Scores from these time points will allow surgeons to quantify outcomes for their individual patients after surgery and also offer insight into each patient's risk of revision.

One limitation of this study is that using revision surgery as an outcome will underestimate the true rate of disease recurrence since not all patients will elect to undergo appropriately indicated revision surgery or revision surgery may be deemed to have minimal impact on improving the recurrent disease. We do not have post-operative endoscopy scores to evaluate this further, and we do not know that time point at which revision surgery was performed. Second, the ESS procedure was not standardi- zed across all surgeons, and there are a proportion of patients who would have received simple polypectomy or very limited ESS. Although surgical variation could affect the revision rate, it would not actually "bias" the data insofar as the overall finding that failure to achieve MCID increases odds of revision surgery. Lastly, as stated above, there is no good evidence to demonstrate that progression to revision surgery can be prevented, although it would seem preferable to identify patients at higher risk for revision surgery to maintain postoperative follow-up and appropriate medical therapies. Despite these limitations, the study is strengthened by the large sample size with complete follow-up data at each of the study time periods. Additionally, the population-based design involving a large number of surgeons with various levels of technical abilities will improve the generalizability of the outcomes.

\section{Conclusion}

Outcomes from this study suggest that failure to improve the SNOT-22 by a minimum of one MCID at 3 months and deterioration of the SNOT-22 score greater than one MCID from the 3 to 12 month postoperative period are both independent risk factors for needing revision ESS with 60 months after primary ESS. In the absence of traditional risk factors for revision ESS, measuring the SNOT-22 score at baseline and then at 3 and 12 months after primary ESS may assist in identifying patients at increased risk for needing revision surgery. 12 months after primary ESS, to detect a symptomatic decline greater than one MCID, may assist surgeons with identifying patients at risk for revision ESS and future research should evaluate if interventions can reduce this risk of revision surgery.

\section{Acknowledgements}

Funding/Support: There was no funding support for this study.

\section{Authorship contribution}

LR: Study concept, data interpretation, manuscript writing, revisions. $\mathrm{CH}$ : Study concept, data analysis, data interpretation, manuscript writing, revisions. ZMS: Study concept, data interpretation, manuscript writing, revisions.

\section{Conflict of interest}

All authors declare that there is no financial interest for the presented research.

\section{References}

1. Bhattacharyya N. Clinical outcomes after revision endoscopic sinus surgery. Arch Oto-HNS 2004; 130:975-978.

2. Litvack JR, Griest S, James KE, Smith TL. Endoscopic and quality-of-life outcomes after revision endoscopic sinus surgery. Laryngoscope 2007; 117:2233-2238.

3. Smith $T L$, Litvack JR, Hwang PHet al. Determinants of outcomes of sinus surgery: a multi-institutional prospective cohort study. Oto-HNS 2010; 142:55-63.
4. Soler ZM, Wittenberg E, Schlosser RJ, Mace JC, Smith TL. Health state utility values in patients undergoing endoscopic sinus surgery. Laryngoscope 2011; 121:2672-2678.

5. Clinger JD, Mace JC, Smith TL. Quality-oflife outcomes following multiple revision 
endoscopic sinus surgery. Int Forum Allergy Rhinol 2012; 2:444-452.

6. Yeung JC, Brandt MG, Franklin JH, Doyle PC, Rotenberg BW, Kilty SJ. Preoperative concerns of patients undergoing endoscopic sinus surgery. Int Forum Allergy Rhinol 2014; 4:658-662.

7. Hopkins C, Browne JP, Slack Ret al. The national comparative audit of surgery for nasal polyposis and chronic rhinosinusitis. Clin Otolaryngol 2006; 31:390-398.

8. Hopkins C, Slack R, Lund V, Brown P, Copley $L$, Browne J. Long-term outcomes from the English national comparative audit of surgery for nasal polyposis and chronic rhinosinusitis. Laryngoscope 2009; 119:2459-2465.

9. Mendelsohn D, Jeremic G, Wright ED, Rotenberg BW. Revision rates after endoscopic sinus surgery: a recurrence analysis. Ann Otol Rhinol Laryngol. 2011; 120:162166.

10. Philpott C, Hopkins C, Erskine Set al. The burden of revision sinonasal surgery in the UK-data from the Chronic Rhinosinusitis Epidemiology Study (CRES): a cross-sectional study. BMJ open 2015; 5:e006680.

11. Senior BA, Kennedy DW, Tanabodee J, Kroger H, Hassab M, Lanza D. Long-term results of functional endoscopic sinus surgery. Laryngoscope 1998; 108:151-157.

12. Naidoo Y, Bassiouni A, Keen M, Wormald PJ. Risk factors and outcomes for primary, revi- sion, and modified Lothrop (Draf III) frontal sinus surgery. Int Forum Allergy Rhinol 2013; 3:412-417.

13. Gore MR, Ebert CS, Jr., Zanation AM, Senior BA. Beyond the "central sinus": radiographic findings in patients undergoing revision functional endoscopic sinus surgery. Int Forum Allergy Rhino 2013; 3:139-146.

14. Van Zele T, Holtappels G, Gevaert P, Bachert C. Differences in initial immunoprofiles between recurrent and nonrecurrent chronic rhinosinusitis with nasal polyps. Am J Rhino Allergy2014; 28:192-198.

15. Zadeh MH, Banthia V, Anand VK, Huang C. Significance of eosinophilia in chronic rhinosinusitis. Am J Rhinol 2002; 16:313-317.

16. Brescia G, Marioni G, Franchella $S$ et al. Can a panel of clinical, laboratory, and pathological variables pinpoint patients with sinonasal polyposis at higher risk of recurrence after surgery? Am J Otolaryngol 2015; 36:554-558.

17. Czerny MS, Namin A, Gratton MA, Antisdel $J$. Histopathological and clinical analysis of chronic rhinosinusitis by subtype. Int Forum Allergy Rhinol 2014; 4:463-469.

18. Soler ZM, Hyer JM, Ramakrishnan Vet al. Identification of chronic rhinosinusitis phenotypes using cluster analysis. Int Forum Allergy Rhino 2015; 5:399-407.

19. Hopkins C, Gillett S, Slack R, Lund VJ, Browne JP. Psychometric validity of the 22-item Sinonasal Outcome Test. Clin Otolaryngol 2009; 34:447-454

20. Rudmik L, Soler ZM, Mace JC, DeConde AS, Schlosser RJ, Smith TL. Using preoperative SNOT-22 score to inform patient decision for Endoscopic sinus surgery. Laryngoscope 2015; 125:1517-1522.

\section{Dr. Luke Rudmik}

Clinical Associate Professor

Division of Otolaryngology -

Head and Neck Surgery

Department of Surgery

University of Calgary

Richmond Road Diagnostic and

Treatment Centre

1820 - Richmond Road SW, T2T 5C7

\section{Calgary}

$\mathrm{AB}$, Canada

Tel: + 1-403-955-8425

Fax: +1-403-210-8435

E-mail:Lukerudmik@gmail.com 